\title{
Vascular cognitive impairment: epidemiology, subtypes, diagnosis and management
}

\author{
SE Black \\ Brill Chair in Neurology, Sunnybrook Health Sciences Centre and University of Toronto; Site Director, Heart and Stroke Foundation Centre \\ for Stroke Recovery; Medical Director, Regional Stroke Program, North \& East Greater Toronto Area; and Director, LC Campbell Cognitive \\ Neurology Research Unit, Toronto, Canada
}

This review is based on a presentation given by Professor Black at the RCPE Care of the Elderly Symposium on 15 September 2010.

ABSTRACT Dementia occurs after stroke in $25 \%$ of patients but also can arise from covert cerebrovascular disease (CVD). 'Silent' lacunes occur in $25 \%$ of the elderly, often associated with focal or confluent hyperintensities on T2-weighted magnetic resonance imaging, which are detected in $95 \%$ of seniors. These covert infarcts predict future stroke and faster cognitive decline. Best practice guidelines advocate screening for cognitive impairment in all phases of overt stroke, when covert CVD is uncovered, when vascular risk factors are present and if patients present with cognitive complaints. Standardised testing is recommended, emphasising executive function and speed of processing. Cholinesterase inhibitors have cognitive enhancing effects in vascular dementia, but the major thrust is still aggressive management of vascular risk factors and healthy lifestyle choices. Given that mixed Alzheimer's dementia and CVD is likely the most common substrate for dementia and that they share common vascular risk factors, a major goal for vascular medicine is cerebrovascular protection, not just to prevent heart attack and stroke, but also to maintain brain health and delay dementia.

KEYWORDS Amyloid angiopathy, cholinesterase inhibitors, cognitive testing, overt and silent stroke, vascular dementia, white matter hyperintensities

DECLARATION OF INTERESTS The author has in the past 12 months received contract research funding from Novartis, Pfizer, Roche and GlaxoSmithKline, speaker's honoraria from Janssen-Ortho, Lundbeck, Eisai and Pfizer and honoraria for ad-hoc consulting from Pfizer, Janssen-Ortho, Novartis, GlaxoSmithKline, Elan, Wyeth Pharmaceuticals and Bristol-Myers Squibb.
Correspondence to SE Black, Sunnybrook Health Sciences Centre, 2075 Bayview Avenue, Room A42 I, Toronto, ON M4N 3M5, Canada

tel. + I 4 I6 480455 I

e-mail sandra.black@sunnybrook.ca

\section{INTRODUCTION}

Vascular cognitive impairment $(\mathrm{VCl})$ often refers to cognitive impairment related to cerebrovascular disease (CVD) that is not severe enough to interfere with autonomy in activities of daily living (ADLs). Just as mild cognitive impairment may be a precursor of Alzheimer's dementia $(A D), \mathrm{VCl}$ may represent an 'at risk' state for decline to vascular dementia $(\mathrm{VaD})$, which may be preventable or delayed by aggressive management of vascular risk factors.' However, $\mathrm{VCl}$ has also been used to embrace any cognitive impairment in which vascular disease is a contributing factor. This would include vascular cognitive impairment-not dementia (VCIND), vascular dementia $(\mathrm{VaD})$ and mixed $\mathrm{VaD}$ and $A D{ }^{2}$ If one adopts this comprehensive concept, $\mathrm{VCl}$ would have comparable prevalence and mortality to so-called 'pure' AD. ${ }^{3}$

The Canadian Study of Health and Aging suggested that, for those over the age of $65, \mathrm{VaD}$ causes $19 \%$ of dementia cases. ${ }^{4}$ However, this was based on history, physical and cognitive testing without the benefit of imaging, and likely overestimated pure $\mathrm{VaD}$, while underestimating mixed $A D$ and $\mathrm{VaD}$. Other epidemiological studies from Western countries suggest that $\mathrm{VaD}$ is the second most common cause of dementia after $A D$, but in Asia it may be the primary aetiology. Like $A D, V a D$ increases with age, with prevalence estimates in people in their 70s ranging from $1.5 \%$ to $4.8 \% .^{5}$ US estimates vary more widely, from $9 \%$ to $39 \%$ of those aged over $65 .{ }^{6}$ Whatever the point estimates, however, clearly all dementias are a major growing health problem as they increase exponentially with age over 65 , and it is projected that two billion of the world's population will be over 65 in a generation.

By general consensus, dementia has usually required a memory deficit plus impairment in at least one other cognitive domain (e.g. language, praxis, visuospatial or executive abilities) that causes loss of autonomy in instrumental and self-care ADLs. ${ }^{7}$ The new Diagnostic and Statistical Manual of Mental Disorders (DSM-V) currently under review suggests the terms 'major neurocognitive disorders' (for dementia) and 'minor neurocognitive disorders' (for CIND or mild cognitive 
impairment), according to the degree to which independence is compromised, and downplays the requirement for memory loss (www.dsm5.org).

Updated NINCDS-ADRDA criteria for AD, ${ }^{8}$ soon to be published, continue to acknowledge gradual onset and progressive memory loss as key, with other cognitive domains becoming increasingly affected over time, but also acknowledge atypical presentations and frequent concomitant pathology such as CVD. The NINDS-AIREN criteria for $\mathrm{VaD}$, which have been most widely used for clinical trials of cognitive enhancers in $\mathrm{VCl}$, specify:

a) cognitive decline in memory and two other domains sufficient to impair functional abilities (attributable to cognitive decline, not just physical disabilities); and

b) evidence for CVD, indicated by focal (i.e. sensorymotor) signs and relevant imaging evidence of stroke. ${ }^{9}$

A temporal relationship between dementia and CVD, e.g. Onset within three months of a stroke or stepwise decline, is required to diagnose 'probable' $\mathrm{VaD}$; otherwise, if criteria a) and b) are met, but there is no abrupt onset or decline, it is called possible VaD. Different criteria for $\mathrm{VaD}$ vary in sensitivity and specificity against pathological verification (e.g. the DSM-IV 0.36 and 0.89 , NINDSAIREN 0.19 and 0.98, and ICD-10 0.19 and 1.00 respectively), so that diagnosis and prevalence can differ markedly depending on the criteria. ${ }^{10}$

\section{HETEROGENEITY OF VCI}

Vascular cognitive impairment is quite a heterogeneous disorder, arising from vascular risk factors alone, such as hypertension, which can drive small vessel disease, manifesting as lacunar infarcts as focal or patchy white matter changes called leukoaraiosis. There can be large vessel infarcts, different types of haemorrhage and hypoperfusion syndromes, including cardiac arrest and postural hypotension. The resulting damage can destroy cognitively eloquent cortical-subcortical structures or it can be confined to the white matter and the deep basal ganglia nuclei, whereby brain circuitry is damaged, potentially interrupting vital large-scale cerebral networks that subtend different behavioural functions."

\section{OVERT CEREBROVASCULAR DISEASE}

Given this heterogeneity, it has become useful to distinguish between what is called overt and covert CVD. Overt disease refers to cognitive impairment associated with a clinically evident stroke event, occurring in approximately $65 \%$ of stroke patients, making it almost as common as sensorimotor manifestations. In about $26-36 \%$, this is severe enough to meet the criteria for dementia (compared with $3 \%$ in age-matched controls). ${ }^{12,13}$ Cognitive impairment increases the likelihood of dependence and long-term placement, and is associated with a higher mortality (6I\% vs $25 \%) .{ }^{12,13}$

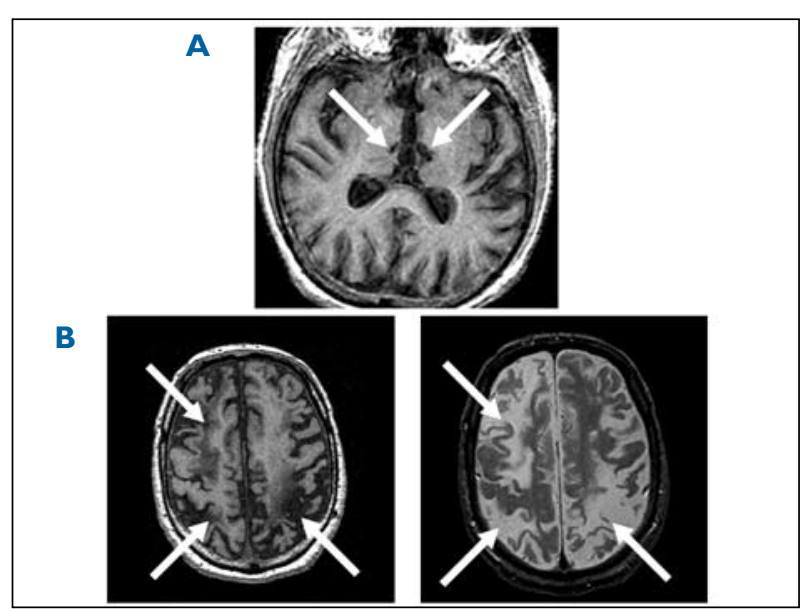

FIGURE I Lesions associated with overt stroke causing pure vascular dementia. A: Small but strategically located infarcts (white arrows) are shown on TI-weighted axial MRI, involving the anterior dorsomedial nucleus of the thalamus bilaterally and causing sudden onset dementia. B: Watershed infarcts related to bilateral carotid occlusions are evident in the right anterior and both posterior brain regions on these high axial MRI slices, TI-weighted in the left panel and T-2 weighted in the right. The damage (not all shown) affected the right dorsolateral frontal region and the bilateral posterior parietal (including the angular gyrus) regions.

Depressive symptoms also occur in $25-50 \%$ of patients after a stroke, and can adversely affect recovery as well as cognition. ${ }^{14,15}$

The probability of suffering post-stroke dementia depends on several factors: the size and location of brain damage, history of previous strokes and imaging evidence of multiple strokes. It is also associated with older age, ethnicity, lower education, vascular risk factors such as diabetes and atrial fibrillation, left-sided lesions and concomitant depression. ${ }^{16}$ With respect to strategic location, damage to the anterior dorsomedial nucleus of the thalamus is particularly notorious as a cause of sudden onset dementia, especially if bilateral (Figure IA). One study suggested that thalamic infarct volume $>55 \mathrm{~mm}^{3}$ represented a threshold for sudden cognitive decline, and that left thalamic infarcts are more likely to cause dementia, whereas right-sided lesions caused VCIND. ${ }^{17}$ Other strategic locations include the angular gyrus, medial frontal region and posterior circulation strokes affecting the hippocampus, particularly if they are bilateral.

More commonly, post-stroke dementia is associated with multiple cortical and/or subcortical infarcts, which can occur simultaneously or in a staged fashion, especially when those infarcts involve cognitively eloquent cortical areas bilaterally. The example illustrated in Figure IB shows bilateral watershed infarcts that caused dementia in a 39-year-old man with bilateral carotid occlusions, who survived 24 years with mild deficits characterised by forgetfulness, word-finding and comprehension difficulties, which prevented him from returning to work, 
but allowed him to maintain good instrumental daily function and self-care activities, play bingo, go shopping, and work as a hospital volunteer. His Mini-Mental State Exam (MMSE) was stable at 23/30. He developed seizures at the age of 60 , which were partially controlled on anticonvulsants, and eventually died from status epilepticus, with a post-mortem confirming the presence of multiple infarcts with no evidence of $A D$.

\section{COVERT CEREBROVASCULAR DISEASE}

The prototypical presentation of post-stroke dementia with sudden onset or stepwise decline is often associated with overt stroke events, verified on imaging, but this is only the tip of the iceberg. An important concept that has been emerging in the field of $\mathrm{VaD}$ is that the CVD contributing to cognitive decline may be otherwise clinically silent. ${ }^{18}$ This insidious form of $\mathrm{VCl}$ arises from covert small vessel pathology, which is very prevalent with ageing, as revealed by large population imaging studies conducted over the past 15 years. Specifically, in individuals over 65, TI-weighted magnetic resonance imaging (MRI) frequently shows small infarcts, defined arbitrarily as lesions of at least $3 \mathrm{~mm}$ in diameter, that are hypointense black holes on $\mathrm{TI}$ and hyperintense foci on proton density/T2-weighted (PD/T2) MRI (Figure 2A). For example, in the Cardiovascular Health Survey of 3,660 individuals, $28 \%$ of seniors, average age 75 , showed silent infarcts. ${ }^{19}$ Their frequency depends on age, with numbers increasing with each decade. ${ }^{20}$ In the Framingham study with mean age $62,12 \%$ had silent infarcts. ${ }^{21}$ In fact, silent strokes may be ten times as prevalent as overt strokes, and in the same Cardiovascular Health Survey, $18 \%$ of those without baseline silent strokes showed new infarcts at five-year follow-up. ${ }^{22}$

Because these lesions are so common, it should not be assumed that they are benign. ${ }^{23}$ As illustrated in the Rotterdam study - a longitudinal study of 1,000 healthy elderly between 60-90 years who were followed for four years - participants with baseline silent infarcts had more rapid cognitive decline, with twice the risk of emergent dementia and five times the risk of stroke (three times the risk after correcting for other vascular risk factors). ${ }^{24}$

It is thought that silent strokes arise mainly from an occlusion of the small penetrating arterioles that plunge in a few centimetres from the surface into the deep white matter from the pial arteries or up into the basal ganglia and thalamus from the middle and posterior cerebral arteries. Hypertension and diabetes, in particular, exacerbate arteriolar disease, causing endothelial damage with increased resistance and decreased autoregulation, ultimately occlusion and obliteration, blood-brain barrier leakage and perivascular space widening.These arterioles also tend to become more tortuous with age, a process accelerated by hypertension. ${ }^{25}$ The result is decreased

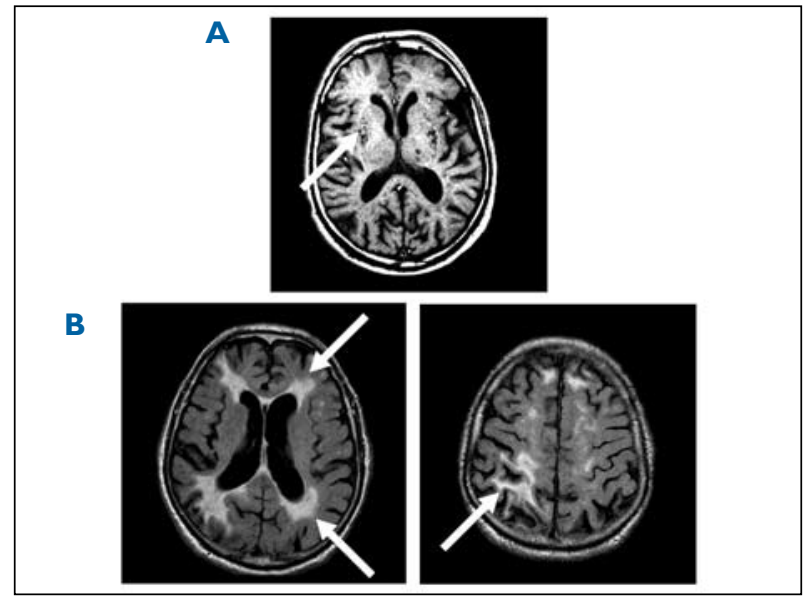

FIGURE 2 Covert small vessel disease. A:White arrow points to a small lacune $3 \mathrm{~mm}$ in diameter that appears as a hypointense black hole on this $\mathrm{TI}$-weighted axial MRI slice. Multiple other lacunes are also visible in the putamen on the right and left and also in the left thalamus. This likely relates to occlusion of arterioles supplying these deep nuclei. B: On this FLAIR axial MRI image, periventricular white matter disease is evident as confluent hyperintensity around the anterior and posterior horns of the lateral ventricles (white arrows in left slice). This may relate to occlusive disease of the deep intramedullary venules. The white arrow on the right side points to white matter infarction in the right parietal lobe.

perfusion in the periventricular white matter regions, which structurally is already relatively hypoperfused, particularly in watershed areas around anterior horns and posterior horns of the lateral ventricles. ${ }^{26}$ This arteriolar vessel disease appears to be a system-wide manifestation of vascular risk factors and ageing affecting other bodily organs, such as the eye (ischaemic optic neuropathy and macular degeneration) and kidney. ${ }^{27}$

However, there is another type of white matter disease that is hyperintense on PD/T2 or fluid attenuation inversion recovery MRIs, often referred to as periventricular or deep white matter hyperintensities (WMH), depending on location. An even more ubiquitous ageing phenomenon than silent strokes, WMH affect the deep intramedullary cerebral venules that drain toward the deep cerebral veins around the ventricles. With human ageing, the deep medullary venules begin gradually to lay down collagen concentrically within the lumen, ${ }^{28}$ possibly in response to chronic hypoxic conditions, modulated by genetic factors. The lumen eventually occludes, resulting in back-up and leakage, causing chronic venous vasogenic oedema in the periventricular regions, particularly around the anterior and posterior horns of the lateral ventricles. ${ }^{18}$ This venous insufficiency leads to poor interstitial fluid circulation in the brain, which may interfere with clearance of toxic amyloid beta fragments along the periarteriolar pathways towards the cortex, possibly exacerbating the pathological amyloid cascade in AD.

In the Cardiovascular Health Survey sample, $95.6 \%$ of $3,30 \mathrm{I}$ healthy volunteers had some degree of $\mathrm{WMH} .{ }^{29}$ In 
the $20 \%$ who had extensive confluent periventricular $\mathrm{WMH}$, cognitive scores were lower and there were problems in gait and balance and dexterity (Figure 2B). Many other studies have likewise shown an association between $\mathrm{WMH}$ and impaired global cognition with slowed information processing and decreased psychomotor speed. ${ }^{30}$ There may be a threshold effect in normal elderly with about $10 \mathrm{cc}$ of periventricular WMH needed to see subtle cognitive effects. ${ }^{31}$

Small vessel disease may be a final common pathway that expresses the adverse effects of vascular risk factors on the brain and may be one of the reasons that $A D$, stroke and $\mathrm{VaD}$ share common vascular risk factors, such as ageing, hypertension, ${ }^{32,33}$ elevated cholesterol, ${ }^{32}$ diabetes, ${ }^{34}$ previous strokes and the apolipoprotein epsilon e4 allele. ${ }^{35}$ Furthermore, stroke and AD commonly co-occur as highlighted by the Nun Study in which 102 sisters aged 76-100 years were prospectively followed cognitively and at autopsy showed that less AD pathology was needed for expression of clinical dementia if infarcts were also present. $^{36}$ In fact, in the 61 sisters who met full pathological criteria for $A D$, only $57 \%$ met clinical criteria for dementia at autopsy. If there were cortical infarcts, $75 \%$ were demented, and if there were concomitant small lacunar strokes, $93 \%$ were demented. Thus if small vessel strokes were present, there was 20 times the risk of dementia. ${ }^{36}$

This has been replicated by other pathological studies which show that mixed $A D$ and $\mathrm{VaD}$ appears to be the most common substrate of dementia. ${ }^{37,38}$ In the British population autopsy study (CFAS) (median age 85), 70\% of subjects had $A D$ and $78 \%$ had cerebrovascular pathology, with small vessel disease being most common (69\%). ${ }^{39}$ In fact, $A D, V a D$ alone and mixed AD plus CVD account for about $80 \%$ of the dementias. To complicate matters further, Lewy body disease may also be co-morbid with $A D$ in elder autopsies, tripling the jeopardy of human brain ageing. ${ }^{40}$

Mixed neuropathologies including vascular degeneration, small vessel damage, haemorrhage and embolic infarcts are prevalent in ageing, making so-called 'pure' AD fairly uncommon in people in their $80 \mathrm{~s}$ and $90 \mathrm{~s}$. Some researchers even suggest that sporadic late-onset Alzheimer's disease itself may be better conceptualised as a vasculopathy, suggesting that the vascular pathology may drive AD." Importantly, AD in turn, through deposition of beta-amyloid $\mathrm{I}-40$ in the capillaries and arterioles, can be the cause of not only occlusive vascular disease but also micro- and macro-haemorrhages.

An illustrative case in point is a 61 -year-old lawyer, who became confused at work, bumping into objects and appearing drunk. He was brought into the emergency department by concerned co-workers, where in the absence of headache, he was found to have a right

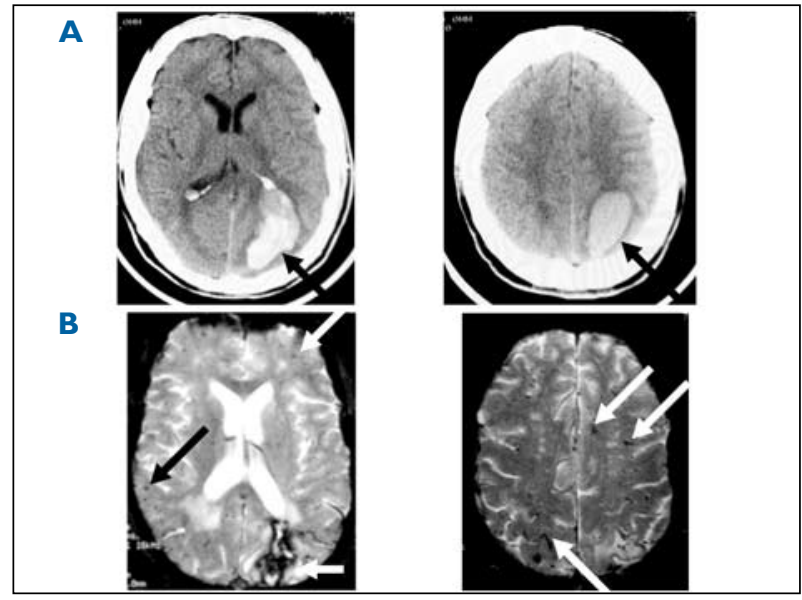

FIGURE 3 Amyloid angiopathy presenting as lobar haemorrhage. A: Left parieto-occipital lobar haemorrhage is seen (black arrows) on admission CT in a 6I-year-old lawyer brought to hospital because he was bumping into furniture and appeared confused. B: Microbleeds, in addition to his residual left posterior lobar haemorrhage (short white arrow), are seen on MRI a few weeks after onset. Gradient echo slices show microbleeds (haemosiderin deposits) in the bilateral frontal and right temporal regions (black and white arrows) including siderosis (curvilinear haemosiderin deposit along a cortical strip) in the right posterior region.

hemianopia, hemineglect, sensory extinction, mild right pronator drift, transcortical sensory aphasia (fluent speech, problems in comprehension, and anomia with intact repetition) as well as alexia, apraxia and Gerstmann's syndrome (finger agnosia, right-left confusion, acalculia and agraphia). His computed tomography (CT) scan (Figure $3 \mathrm{~A}$ ) showed a large left parietal-occipital lobar haemorrhage. There was no secondary cause found on angiography. A follow-up gradient echo T2* MRI sequence a few weeks later showed multiple microbleeds with haemosiderin deposits, not only at the site of the overt haemorrhage but scattered throughout the brain (Figure 3B). He was discharged to rehabilitation, where his apraxia and aphasia improved, but difficulties in reading, mental calculations and his right visual field deficit persisted. The patient had to give up his job as a successful corporate lawyer and could no longer drive, but managed his ADLs.

He was lost to follow-up until seven years later when he returned to the clinic because of gradual cognitive decline and severe short-term memory problems. His MMSE at this time was $16 / 30$, but repeat MRI showed no major change except for more diffuse cerebral atrophy. After some initial stabilisation on a cholinesterase inhibitor, he showed a gradual progressive decline, and was placed in a nursing home where, nine years after his initial haemorrhage, he was found in his bed without vital signs. Autopsy confirmed the old haemorrhages and multiple microbleeds from amyloid angiopathy and stage 5/6 AD by Braak and Braak criteria. ${ }^{42}$ 
Amyloid angiopathy, manifest as cerebral microbleeds, on standard gradient echo on I.5T T2* MRI, was evident in $29 \%$ of AD patients in one memory clinic sample, with multiple bleeds noted in $48 \%$ of the AD group, compared to $12 \%$ in normal controls (multiple in $33 \%$ ). ${ }^{43}$ Amyloid positron emission tomography imaging detects fibrillar amyloid whether deposited in plaques or around vessels, but there appears to be an occipital predominance in amyloid uptake in patients presenting with lobar haemorrhage from cerebral amyloid angiopathy (CAA) ${ }^{44}$ In current clinical trials of monoclonal antibodies and vaccines as potential disease-modifying therapies for $A D$, cerebral microbleeds are screened for on gradient echo MRI to determine eligibility and for monitoring safety. A gradient echo sequence, preferably $5 \mathrm{~mm}$ or less slice thickness at $1.5 \mathrm{~T}$ takes about five minutes to acquire and should be included when using MRI to work up dementia and also for stroke. Location in the deep nuclei or brainstem suggests hypertensive aetiology, but lobar microbleeds are usually related to $C A A$ from $A D$ (assuming vascular malformations are ruled out by MR or CT angiography). ${ }^{45}$

Microbleeds can present a diagnostic dilemma for clinicians managing elderly subjects with vascular risk factors. Their presence may increase risk of cerebral bleeding with the use of antiplatelet agents or anticoagulants. However, the evidence is still somewhat conflicting on this risk, and if there is an important need for vascular protection such as coronary artery disease or previous ischaemic stroke, it is recommended that antithrombotics be used with caution..$^{45}$ The use of anticoagulants may be more problematic, so the risks should be carefully weighed. The case of the 61-year-old lawyer highlights the grim vascular manifestations of Alzheimer's pathology and underlines that $A D$ deserves to be included as a risk factor for stroke. Alzheimer's dementia can cause cerebral haemorrhage and small vessel infarction and, as part of this vicious cycle, ischaemic damage and haemorrhagic stroke can exacerbate the development and clinical expression of $A D$.

Given the prevalence and importance of vascular cognitive impairment, new best practice stroke guidelines have suggested that cognitive screening should be performed in any individuals considered at high risk for cognitive and perceptual impairments related to vascular disease. ${ }^{46}$ This would include individuals with hypertension, hyperlipidaemia, diabetes, age over 65 , history of clinical stroke or neuroimaging findings suggesting covert lacunar or white matter disease, damage to other target organs such as kidneys and eyes, and vascular patients who present with complaints of cognitive and functional decline as the primary cause of referral or as reported during history taking (www.strokebestpractices.ca). ${ }^{46}$

In 2005, there was an international consensus conference sponsored by the National Institutes of Health and
Canadian Stroke Network to harmonise and standardise clinical, cognitive and behavioural assessment, imaging, neuropathological examination and even experimental models of $\mathrm{VCl} .{ }^{47}$ The neuropsychological working group was asked to recommend 60-minute, 30-minute and five-minute protocols. It emphasised executive activation (timed tasks) and strategic memory processes, but included brief tests of naming and complex figure copying, and standardised mood and behaviour questionnaires. The five-minute protocol comprised fiveword registration, recall and recognition, a six-item orientation task and phonemic fluency, in which the subject is asked to generate as many words as possible in one minute beginning with a letter (e.g. 'F').

The group also endorsed the Montreal Cognitive Assessment (MoCA), a 10-15-minute test that probes executive function better than the traditional MMSE, which was developed to discriminate dementia cases from other mental illnesses. The MoCA successfully discriminated mild cognitive impairment from agematched controls with a specificity of $81 \%$ and a sensitivity of $80 \% .{ }^{49}$ It is freely downloadable from the internet (www.mocatest.org), translated into more than 25 languages, available in multiple forms and is being used in the Alzheimer's Disease Neurolmaging extension trial (ADNI-2). It was also endorsed by the best practice Canadian guidelines mentioned above, and is being used in many stroke prevention clinics, as well as for cognitive assessment in other disorders such as Parkinson's disease and multiple sclerosis.

Small vessel disease as a primary cause of dementia can present as a distinctive syndrome called subcortical ischaemic vascular dementia (SIVD). ${ }^{50}$ It may gradually develop in a manner that mimics $A D$, but memory complaints are less prominent and, although patients will show impaired short-term memory retrieval (i.e. spontaneous recall), their encoding is relatively intact: there is less severe forgetting, they benefit from cueing and recognition memory is relatively intact. The most prominent difficulties are with executive function tasks, thought to reflect disruption in subcortical circuitry interconnecting posterior and subcortical brain regions with the frontal lobes. This manifests as impaired goal formulation, initiation, planning, organising, sequencing activities, abstraction and conceptualisation, focused and sustained attention, as well as carrying out goal-directed behaviour and the ability to shift from one task to another.

Other early clinical features include gait disorder and poor balance, and sometimes urinary frequency and incontinence. Some patients may show problems with dysarthria and dysphagia and demonstrate extrapyramidal signs suggestive of Parkinsonism with bradykinesia and mild rigidity. Bilateral pyramidal tract injury from lacunes in the internal capsules of both hemispheres, can cause pseudobulbar palsy, a feature of 
A

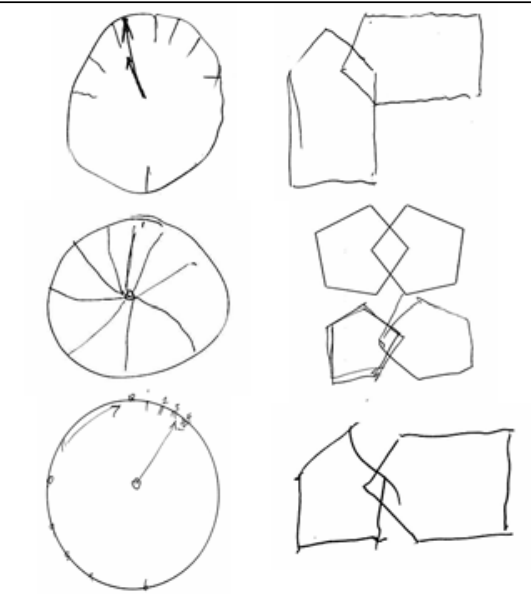

FIGURE 4 Abnormal clock drawings in $\mathrm{VCl}$ patients with normal Mini Mental State Exam (MMSE) scores who were asked to draw a clock and set the hands at 10 past II. A: 81 -year-old man with 23 years of education and MMSE score of 27/30, diagnosed with mixed Alzheimer's dementia (AD) and cerebrovascular disease (CVD). B: 78-year-old woman with 12 years of education with an MMSE score of 25/30, diagnosed with mixed AD and CVD. C: 75 -year-old woman with 16 years of education scoring $26 / 30$ on the MMSE, diagnosed with probable vascular dementia.

which, in addition to dysarthria, dysphagia and facial spasticity, may be emotional incontinence, whereby the patient easily cries or laughs, perhaps triggered by a social situation, but far in excess of what the person actually feels. This can be ameliorated by selective serotonin re-uptake inhibitors.

Subcorticol ischaemic vascular dementia can also be associated with problem behaviours including marked apathy and depression, stubbornness and irritability. Patients may perform relatively well on the MMSE, which is weighted for language and memory abilities and can be relatively preserved in SIVD. However, clock drawing can bring out difficulties, particularly in placement of the hands, such as indicating $1 \mathrm{I}: 10$. To do this properly requires abstraction (recognising that the minute hand must point to the two and be longer than the hour hand), proper spacing of the numbers and placement of the minute and hour hands (Figure 4).

\section{COGNITIVE ENHANCEMENT}

Evidence suggests that cholinergic markers can be decreased in patients with $\mathrm{VaD}^{51,52}$ and some recent studies have shown that subcortical ischaemic WMH often affects the cortical cholinergic projections emanating from the nucleus basalis of Meynert. ${ }^{53}$ In fact, in a study of $60 \mathrm{AD}$ patients, a rating scale for WMH involvement of the cholinergic pathways correlated better with executive function than overall WMH load. ${ }^{54}$ Given that pivotal trials demonstrated some benefits of cholinesterase inhibitors in $A D$, clinical trials were undertaken to investigate safety and efficacy in $\mathrm{VaD}$.
Briefly, in the galantamine trial, which included both $\mathrm{VaD}$ ( $40 \%)$, patients with possible $A D$ related to vascular disease, and possible $\mathrm{VaD}$, there was less decline in cognition, global function and ADLs over a six-month period in 396 patients treated with galantamine compared with 196 controls. ${ }^{55}$ The pure $\mathrm{VaD}$ subgroup, in whom the placebo group did not decline over six months, showed improvement in cognition, but not in global or daily function. A larger galantamine study targeting 'pure' $\mathrm{VaD}$ patients $(\mathrm{n}=788)$ achieved significance for cognitive improvement, a trend for global functioning, but no difference in ADL measures. ${ }^{56}$

Two parallel trials of donepezil in patients with $\mathrm{VaD}$, in which patients with clinical features suggesting $A D$ were excluded, demonstrated consistent modest improvement on the cognitive measures, compared with the placebo group, who did not decline over six months, but effects on global function and ADLs were less consistent. ${ }^{57,58}$ A problem with assessing function in this population is that concomitant physical deficits associated with stroke often confound determination that daily function changes are only attributable to cognitive factors. In a combined analysis of the two donepezil studies $(n=1,219)$, there were modest but significant benefits in cognition, global and daily functioning, particularly in instrumental ADLs, which generally correlate with executive functions. ${ }^{60}$

In a third large six-month trial of donepezil in $\mathrm{VaD}$ $(n=974)$, there were again modest beneficial effects on cognition but not on global or ADL function. ${ }^{60}$ Interestingly, post-hoc analysis of the subgroup with hippocampal atrophy using a centralised qualitative rating on baseline $C T$ and MRI scans showed cognitive stabilisation with treatment versus decline in the placebo group, whereas in those without atrophy, cognition improved with treatment and was stable on placebo. This suggests that hippocampal atrophy may need to be taken into account when assessing response to cholinesterase inhibitors in $\mathrm{VaD}^{60}$ Overall adverse event rates were similar. No deaths occurred in the placebo group, which was less than expected, and II deaths occurred in the donepezil group, a death rate (I.7\%) similar to both treatment groups in the previous donepezil trials in $\mathrm{VaD}$.

Clinical trials of memantine in $\mathrm{VaD}$ also showed cognitive benefit, but global and functional scores did not differ from placebo. ${ }^{61,62}$ Of note, most of these $\mathrm{VaD}$ studies were randomised, double-blind, placebo-controlled sixmonth trials, only some of which had centralised neuroimaging evaluation for relevant CVD. However, they all used the Alzheimer's Disease Assessment Scale (ADAS-Cog), which is relatively insensitive to executive function as a primary outcome measure, even though additional executive tasks were added in some trials (called the VADAS-Cog). The difficulty of evaluating daily function outcomes in the presence of physical disability from stroke also remains a challenge to $\mathrm{VaD}$ trials. 


\section{CONCLUSIONS}

In summary, best practice suggests screening for $\mathrm{VCl}$ in all phases of overt stroke disease in which a quarter of patients are at risk of dementia, but also when silent stroke disease is uncovered, and even if vascular risk factors are present, including age and evidence of organ damage in other target organs. Executive functioning and speed of processing are important to assess in patients with $\mathrm{VCl}$. Standardised testing is recommended, for example, the MoCA for a quick screen because it is more weighted for executive function. More detailed standardised assessment tools have also been suggested.

Of note are population autopsy studies suggesting that mixed Alzheimer's and cerebrovascular disease is the most common substrate for dementia, especially in those older than 75 years. Small vessel disease is ubiquitous in our ageing population, not only in the brain but also in the body, and often co-exists with Alzheimer's disease. It is not benign and represents an important risk factor for future overt stroke, for gait problems and falls, for urinary incontinence and for cognitive and behavioural decline. The cognitive enhancing agents can modestly enhance cognition in $\mathrm{VaD}$, based on pivotal trials, but functional benefits are unproven and whether cognitive

\section{REFERENCES}

I Hachinski VC. Multi-infarct dementia: a reappraisal. Alzheimer Dis Assoc Disord 1991; 5:64-8. doi:10.1097/00002093-199100520-00002

2 O'Brien JT, Erkinjuntti T, Reisberg B et al. Vascular cognitive impairment. Lancet Neurol 2003; 2:89-98. doi:I0.10I6/SI4744422(03)00305-3

3 Rockwood K, Wentzel C, HachinskiV et al. Prevalence and outcomes of vascular cognitive impairment. Vascular Cognitive Impairment Investigators of the Canadian Study of Health and Aging. Neurology 2000; 54:447-5I.

4 Canadian Study of Health and Aging Working Group. Canadian study of health and aging: study methods and prevalence of dementia. CMAJ 1994; 150:899-913.

5 Rocca WA, Hofman A, Brayne C et al. The prevalence of vascular dementia in Europe: facts and fragments from 1980-1990 studies. Ann Neurol 1991; 30:817-24. doi:10.1002/ana.4103006II

6 Kase CS, Wolf PA, Chodosh EH et al. Prevalence of silent stroke in patients presenting with initial stroke: the Framingham Study. Stroke 1989; 20:850-2.

7 American Psychiatric Association. Diagnostic and statistical manual of mental disorders. 4th ed. Washington DC: American Psychiatric Association; 1994.

8 McKhann G, Drachman D, Folstein M et al. Clinical diagnosis of Alzheimer's disease: Report of the NINCDS-ADRDA work group under the auspices of Department of Health and Human Services Task Force on Alzheimer's disease. Neurology 1984; 34:939-44.

9 Román GC, Tatemichi TK, Erkinjuntti T et al. Vascular dementia: Diagnostic criteria for research studies. Report of the NINDSAIREN International Workshop. Neurology 1993; 43:250-60.

10 Gold G, Bouras C, Canuto A et al. Clinicopathological validation study of four sets of clinical criteria for vascular dementia. Am J Psychiatry 2002; I59:82-7. doi:I0.I I76/appi.ajp. I59.I.82

I I Greicius MD, Srivastava G, Reiss AL et al. Default-mode network activity distinguishes Alzheimer's disease from healthy aging: evidence from functional MRI. Proc Natl Acad Sci U S A 2004; I01:4637-42. doi:I0.1073/pnas.0308627I01 benefits are sustained in the longer term has not been evaluated. Potential side effects and benefits must be weighed as well as costs and, importantly, these agents have not been approved by most regulatory agencies for use in this condition, so their use remains off-label and is not usually covered by governmental healthcare formularies.

A major conclusion from the research of the past 20 years in $\mathrm{VCl}$ is that a significant goal for vascular medicine is vascular risk factor control, not just to prevent heart attack and stroke, but also dementia. In addition to appropriate, evidence-based use of pharmacotherapy, ideally customised to each individual, public health measures are important, including efforts to reduce obesity, increase exercise, encourage heart-healthy eating (emphasising high fibre and reduction in dietary cholesterol and salt) and other healthy lifestyle choices in every age group.

Acknowledgments The author is grateful to the Canadian Institutes of Health Research for operating funds (MTI3129) and for salary support from the Departments of Medicine (Neurology) and Brill Chair in Neurology from Sunnybrook Health Sciences Centre and the University of Toronto, the Brain Sciences Research Program at the Sunnybrook Research Institute and the Heart and Stroke Foundation Centre for Stroke Recovery.

I2 Tatemichi TK, Desmond DW, Mayeux R et al. Dementia after stroke: Baseline frequency, risks and clinical features in a hospitalized cohort. Neurology 1992; 42: I I85-93.

13 Desmond DW, Moroney JT, Sano $M$ et al. Incidence of dementia after ischemic stroke: results of a longitudinal study. Stroke 2002; 33:2254-60. doi:10.I I6I/0I.STR.0000028235.9I778.95

14 Pohjasvaara T, Leppävuori A, Siira I et al. Frequency and clinical determinants of poststroke depression. Stroke 1998; 29:23 II-7.

I5 Herrmann N, Black SE, Lawrence J et al. The Sunnybrook Stroke Study: a prospective study of depressive symptoms and functional outcome. Stroke 1998; 29:618-24.

16 Pohjasvaara T, Erkinjuntti T, Vataja R et al. Comparison of stroke features and disability in daily life in patients with ischemic stroke aged 55 to 70 and 71 to 85 years. Stroke 1997; 28:729-35.

17 Swartz RH, Black SE. Anterior-medial thalamic lesions in dementia: frequent, and volume dependently associated with sudden cognitive decline. J Neurol Neurosurg Psychiatry 2006; 77:1307-12. doi:I0.I I36/jnnp.2006.09|56 I

I8 Black S, Gao F, Bilbao J. Understanding white matter disease: imaging-pathological correlations in vascular cognitive impairment. Stroke 2009; 40:S48-S52. doi:I0.I I6I/STROKEAHA. 108.537704

19 Longstreth WT Jr, Bernick C, Manolio TA et al. Lacunar infarcts defined by magnetic resonance imaging of 3660 elderly people: the Cardiovascular Health Study. Arch Neurol 1998; 55:1217-25. doi:I0.100I/archneur.55.9.1217

20 Vermeer SE, Longstreth WT Jr, Koudstaal PJ. Silent brain infarcts: a systematic review. Lancet Neurol 2007; 6:611-9. doi:10.10161 SI474-4422(07)70I70-9

21 DeCarli C, Massaro J, Harvey D et al. Measures of brain morphology and infarction in the Framingham Heart Study: establishing what is normal. Neurobiol Aging 2005; 26:49I-5I0. doi: 10.1016/j.neurobiolaging.2004.05.004

22 Longstreth WT Jr, Dulberg C, Manolio TA et al. Incidence, manifestations, and predictors of brain infarcts defined by serial cranial magnetic resonance imaging in the elderly: the 
Cardiovascular Health Study. Stroke 2002; 33:2376-82. doi:10.1 I6I/0I.STR.000003224I.58727.49

23 Bernick C, Kuller L, Dulberg $C$ et al. Silent MRI infarcts and the risk of future stroke: the Cardiovascular Health Study. Neurology 200I; 9; 57:1222-9.

24 Vermeer SE, den Heijer T, Koudstaal PJ et al. Incidence and risk factors of silent brain infarcts in the population-based Rotterdam Scan Study Stroke 2003; 34:392-6. doi:I0.1I6I/0I.STR.000005263I.98405.15

25 Thore CR, Anstrom JA, Moody DM et al. Morphometric analysis of arteriolar tortuosity in human cerebral white matter of preterm, young, and aged subjects. J Neuropathol Exp Neurol 2007; 66: 337-45. doi: 10.1097/nen.0b013e3180537/47

26 Holland CM, Smith EE, Csapo I et al. Spatial distribution of whitematter hyperintensities in Alzheimer disease, cerebral amyloid angiopathy, and healthy aging. Stroke 2008; 39:1 | 27-33. doi: | $0.1 \mid$ |6I| STROKEAHA. I07.497438

27 Thompson CS, Hakim AM. Living beyond our physiological means: small vessel disease of the brain is an expression of a systemic failure in arteriolar function: a unifying hypothesis. Stroke 2009; 40:e322-e330. doi: I0.1 I6I/STROKEAHA. 108.542266

28 Moody DM, Brown WR, Challa VR et al. Periventricular venous collagenosis: association with leukoaraiosis. Radiology 1995; 194:469-76.

29 Longstreth WT, Manolio TA, Arnold AM et al. Clinical correlates of white matter findings on cranial magnetic resonance imaging of 330 I elderly people: the Cardiovascular Health Study. Stroke 1996 27:1274-82.

30 De Groot JC, de Leeuw FE, Oudkerk M et al. Cerebral white matter lesions and cognitive function: the Rotterdam Scan Study. Ann Neurol 2000; 47:| 145-5I. doi:10.1002//53I-8249(200002)47:2<|45::AIDANA3>3.0.CO;2-P

3I DeCarli C, Murphy DGM, Tranh M et al.The effect of white matter hyperintensity volume on brain structure, cognitive performance, and cerebral metabolism of glucose in 51 healthy adults. Neurology 1995; 45:2077-84.

32 Kivipelto M, Helkala EL, Laakso MP et al. Midlife vascular risk factors and Alzheimer's disease in later life: longitudinal, population based study. BMJ 200 I; 322: 1447-5I. doi: I 0.I I 36/bmj.322.7300.1447

33 Launer LJ, Ross GW, Petrovitch $\mathrm{H}$ et al. Midlife blood pressure and dementia: the Honolulu-Asia aging study. Neurobiol Aging 2000; 21:49-55. doi: $10.1016 / 50197-4580(00) 00096-8$

34 Arvanitakis Z,Wilson RS, Bienias JL et al. Diabetes mellitus and risk of Alzheimer disease and decline in cognitive function. Arch Neurol 2004 61:661-6. doi:10.100I/archneur.61.5.661

35 Slooter AJ, Cruts M, Kalmijn S et al. Risk estimates of dementia by apolipoprotein $\mathrm{E}$ genotypes from a population-based incidence study: the Rotterdam Study. Arch Neurol 1998; 55:964-8. doi:10.100I/ archneur.55.7.964

36 Snowdon DA, Greiner LH, Mortimer JA et al. Brain infarction and the clinical expression of Alzheimer disease.The Nun Study. JAMA 1997; 277:8I3-7.

37 Schneider JA, Arvanitakis Z, Bang W et al. Mixed brain pathologies account for most dementia cases in community-dwelling older persons. Neurology 2007; 69:2197-204. doi:10.1212/01. wnl.000027/090.28I48.24

38 Lim A, Tsuang D, Kukull W et al. Clinico-neuropathological correlation of Alzheimer's disease in a community-based case series. J Am Geriatr Soc 1999; 47:564-9.

39 Neuropathology Group of the Medical Research Council Cognitive Function and Ageing Study (MRC CFAS). Pathological correlates of late-onset dementia in a multicentre, community-based population in England and Wales. Lancet 200I; 357:169-75. doi: 10.1016/501406736(00)03589-3

40 Hansen LA, Samuel W. Criteria for Alzheimer's disease and the nosology of dementia with Lewy bodies. Neurology 1997; 48: I26-32.

4I De la Torre JC. Alzheimer disease as a vascular disorder: nosological evidence. Stroke 2002; 33:1152-62. doi:10.1/61/0I. STR.00000I442I.15948.67

42 Braak H, Braak E. Neuropathological stageing of Alzheimer-related changes. Acta Neuropathol 1991; 82:239-59. doi:10.1007/BF00308809
43 Pettersen JA, Sathiyamoorthy G, Gao FQ et al. Microbleed topography, leukoaraiosis, and cognition in probable Alzheimer disease from the Sunnybrook Dementia Study. Arch Neurol 2008;65:790-5. doi:I0.1001/archneur.65.6.790

44 Johnson KA, Gregas M, Becker JA et al. Imaging of amyloid burden and distribution in cerebral amyloid angiopathy. Ann Neurol 2007; 62:229-34. doi:10.1002/ana.2II64

45 Cordonnier C. Brain microbleeds. Pract Neurol 2010;10:94-100. doi:I0.I I36/jnnp.2010.206086

46 Lindsay P, Bayley M, McDonald A et al. Toward a more effective approach to stroke: Canadian best practice recommendations for stroke care. CMAJ 2008; 20; I78: I4I8-25.

47 Hachinski V, ladecola C, Petersen RC et al. National Institute of Neurological Disorders and Stroke-Canadian Stroke Network vascular cognitive impairment harmonization standards. Stroke 2006; 37:2220-4I. doi:I0.II6I/0I.STR.0000237236.88823.47

48 Folstein MF, Folstein SE, McHugh PR. 'Mini Mental State': a practical method for grading the cognitive state of patients for the clinician. J Psychiatr Res 1975; 12:189-98. doi:10.1016/0022-3956(75)90026-6

49 Nasreddine ZS, Phillips NA, Bedirian V et al. The Montreal Cognitive Assessment, MoCA: a brief screening tool for mild cognitive impairment. J Am Geriatr Soc 2005; 53:695-9. doi:I0.IIIIII i.I532-54I5.2005.5322I.x

50 Erkinjuntti T. Subcortical ischemic vascular disease and dementia. Int Psychogeriatr 2003;15 (Suppl I):23-6. doi:10.1017/ SI04I6I0203008925

51 Kalaria RN, Ballard C. Overlap between pathology of Alzheimer disease and vascular dementia. Alzheimer Dis Assoc Disord 1999; I3:SI I5-SI23. doi:I0.1097/00002093-1999I2003-000I7

52 Perry E, Ziabreva I, Perry R et al. Absence of cholinergic deficits in 'pure' vascular dementia. Neurology 2005; 64:I32-3.

53 Swartz RH, Sahlas DJ, Black SE. Strategic involvement of cholinergic pathways and executive dysfunction: does location of white matter signal hyperintensities matter? J Stroke Cerebrovasc Dis 2003; 12:29-36. doi: 10.1053/jscd.2003.5

54 Bocti C, Swartz RH, Gao FQ et al. A new visual rating scale to assess strategic white matter hyperintensities within cholinergic pathways in dementia. Stroke 2005; 36:2I26-3I. doi:I0.1I6I/0I. STR.0000183615.07936.b6

55 Erkinjuntti T, Kurz A, Gauthier S et al. Efficacy of galantamine in probable vascular dementia and Alzheimer's disease combined with cerebrovascular disease: a randomised trial. Lancet 2002; 359: I283-90. doi:I0.1016/S0140-6736(02)08267-3

56 Auchus AP, Brashear HR, Salloway S et al. Galantamine treatment of vascular dementia: a randomised trial. Neurology 2007; 69:44858. doi:I0.12 I2/0I.wnl.0000266625.316I5.f6

57 Black S, Román GC, Geldmacher DS et al. Efficacy and tolerability of donepezil in vascular dementia: positive results of a 24-week, multicenter, international, randomized, placebo-controlled clinical trial. Stroke 2003; 34:2323-30. doi:I0.II6I/0I. STR.000009I396.95360.EI

58 Wilkinson D, Doody R, Helme $R$ et al. Donepezil in vascular dementia: a randomized, placebo-controlled study. Neurology 2003; 6l:479-86.

59 Román GC, Wilkinson DG, Doody RS et al. Donepezil in vascular dementia: combined analysis of two large-scale clinical trials. Dement Geriatr Cogn Disord 2005; 20:338-44. doi:I0.I I59/000088494

60 Román GC, Salloway S, Black SE et al. Randomized, placebocontrolled, clinical trial of donepezil in vascular dementia. Differential effects by hippocampal size. Stroke 2010; 41:1213-21. doi:I0.I I6I/STROKEAHA.109.570077

6I Wilcock G, Mobius HJ, Stoffler A. A double-blind, placebocontrolled multicentre study of memantine in mild to moderate vascular dementia (MMM500). Int Clin Psychopharmacol 2002; 17:297-305. doi:I0.1097/00004850-2002II000-00005

62 Orgogozo JM, Rigaud AS, Stoffler A et al. Efficacy and safety of memantine in patients with mild to moderate vascular dementia: a randomized, placebo-controlled trial (MMM 300). Stroke 2002; 33:I834-9. doi:I0.I I6I/0I.STR.0000020094.08790.49 